\title{
Cuantificación del desperdicio de alimentos en servicios de alimentación de la Universidad de Costa Rica
}

DOI: 10.17533/udea.penh.v23n2a02

PERSPECTIV

PERSPECTIVAS EN NUTRICIÓN HUMANA

ISSN $01244-4108$

Escuela de Nutrición y Dietética, Universidad de Antioquia. Medellín, Colombia

Vol. 23, N. ${ }^{\mathrm{p}} 2$, julio-diciembre de 2021, pp. 143-157.

Artículo recibido: 23 de febrero de 2021

Aprobado: 24 de junio de 2021

\section{Cindy Hidalgo-Víquez*; Marcela Peña-Vásquez²}

\section{- - -Resumen}

Antecedentes: los alimentos desperdiciados en ventas al detalle en Latinoamérica (15\%) podrían suplir las necesidades alimenticias del $64 \%$ de quienes sufren hambre en la región. Objetivo: determinar los desperdicios de alimentos en tres servicios de alimentación de la sede Rodrigo Facio de la Universidad de Costa Rica a partir de la guía de medición de la Red Costarricense para Disminuir la Pérdida y el Desperdicio de Alimentos. Materiales y métodos: los desperdicios en los servicios de alimentación N1 y N2 fueron medidos en 2018 y 2019 y en el N3 en 2019; se realizaron 15 mediciones siguiendo la metodología de la guía y se reportaron como porcentaje del total de producción de sólidos. Resultados: los desperdicios en la producción total fueron 15,0-16,6 \% en 2018 y 6,4-11,5\% en 2019. No se encontraron diferencias entre los servicios de alimentación en la producción promedio ( $p=0,1467)$, en el desperdicio del área de almacenamiento $(p=0,1293)$ ni en la producción de alimentos $(p=0,7718)$. Se encontraron diferencias en el desperdicio entre los servicios de alimentación en el área de servicio: desperdicios en barra $(p=0,0197)$, en plato/ desayuno $(p=0,0075)$ y en plato/almuerzo $(p=0,0362)$. Conclusiones: la cantidad de desperdicios encontrados son menores a los reportados en otros estudios.

Palabras clave: desperdicio de alimentos, restaurante, medición, servicios de alimentación, residuo de alimentos, residuos de la preparación de alimentos, restos de comida, sobras de alimentos, sobras de comida.

$1^{*}$ Autor de correspondencia. Licenciada, docente e investigadora. Escuela de Nutrición, Universidad de Costa Rica. Montes de Oca, San José, Costa Rica. cindy.hidalgoviquez@ucr.ac.cr. https://orcid.org/0000-0002-0269-5008

2 Magíster, nutricionista. Supervisora del área nutricional de los contratos de servicio de alimentación. Oficina de Servicios Generales, Sección Gestión de Servicios Contratados, Universidad de Costa Rica. Montes de Oca, San José, Costa Rica. marcela.penavasquez@ucr.ac.cr. https://orcid.org/0000-0002-9236-7656

Cómo citar este artículo: Hidalgo-Víquez C, Peña-Vásquez M. Cuantificación del desperdicio de alimentos en servicios de alimentación de la Universidad de Costa Rica. Perspect Nutr Humana. 2021;23:143-57. DOI: 10.17533/udea.penh.v23n2a02 


\section{Quantification of Food Waste in Food Services of the Universidad de Costa Rica}

\section{Abstract}

Background: Food wasted in retail in Latinamerica (15\%) could supply the nutritional needs of $64 \%$ of the hungry in the region. Objective: To determine food waste in three food services of the Rodrigo Facio campus of the Universidad de Costa Rica, using the measurement guide of the Costa Rican Network to reduce food loss and waste. Materials and Methods: Waste in food services N1 and N2 were measured in 2018 and 2019, and in the N3 in 2019. In total 15 measurements were made following the methodology of the guide and they were reported as a percentage of total solids production. Results: Waste in total production was 15.0-16.6\% in 2018 and $6.4-11.5 \%$ in 2019. No differences were found between food services in average production ( $p=0.1467)$, in the waste storage area $(p=0.1293)$, nor food production $(p=0.7718)$. Differences in waste were found between food services in the service area: Waste in the service bar $(p=0.0197)$, in plate/breakfast $(p=0.0075)$, and in plate/lunch $(p=0.0362)$. Conclusions: The amount of waste found is lower than that reported in other studies.

Keywords: Food waste, restaurant, measurement, food services, food residues, food preparation waste, leftovers from meals, refuse from food preparation.

\section{INTRODUCCIÓN}

El problema mundial de la alimentación se ha trabajado desde varios ejes, uno de los cuales ha sido la pérdida y desperdicio de alimentos (PDA), reconociendo la relación que puede tener este tema en la seguridad alimentaria y nutricional y el impacto en varios de los objetivos de desarrollo, como el de reducción del hambre y producción y consumo responsable (1). Además, el impacto ambiental relacionado con el hecho de que alrededor de una tercera parte de los alimentos que se producen a nivel mundial se pierden o desperdician $(2,3)$.

Se conoce como desperdicio de alimentos cuando un alimento es desechado al final de la cadena alimentaria, por ejemplo, en la venta minorista y consumo final y está relacionado con el comportamiento de los vendedores minoristas y los consumidores finales (4).

Los servicios de alimentación (SA) representan una fuente importante de desperdicio de alimentos (5).
En Europa, se ha estimado que se desperdician aproximadamente $21 \mathrm{~kg}$ por persona por año en cada SA, lo que representa un desperdicio aproximado de 10,5 millones de toneladas en este sector (6).

En Latinoamérica, se ha estimado que cada año la región desperdicia al menos el $15 \%$ de sus alimentos disponibles. El desperdicio por segmento es un $28 \%$ para el consumo, $28 \%$ en producción, $22 \%$ en manejo y almacenamiento, $17 \%$ para la distribución y el mercado y $6 \%$ durante el procesamiento. Los alimentos que se desperdician a nivel de la venta al detalle en la región podrían satisfacer las necesidades alimenticias de más de 30 millones de personas, es decir, el $64 \%$ de quienes sufren hambre en la región (7).

En Costa Rica, desde el año 2014 se conformó la Red Costarricense para Disminuir la PDA (8), la cual ha trabajado en coordinación con la Red Costarricense de Instituciones Educativas Sostenibles (REDIES) creada en 2009 (9). La Universidad de Costa Rica (UCR), como miembro activo de estas 
dos redes, se ha comprometido con la reducción de la PDA mediante la ejecución de diferentes investigaciones que tienden a la cuantificación de dichas pérdidas en las diferentes etapas del procesamiento de alimentos en los SA, para poder definir acciones efectivas en su disminución (10).

El objetivo de esta investigación fue determinar las pérdidas de alimentos según áreas de medición, en tres SA de la sede Rodrigo Facio de la Universidad de Costa Rica, según la guía de medición de la Red Costarricense para Disminuir la PDA.

\section{MATERIALES Y MÉTODOS}

La UCR es uno de los centros de educación superior pública más importantes de Costa Rica. La Universidad tiene ocho sedes y cuatro recintos en todo el territorio nacional, de los cuales la más grande es la Sede Rodrigo Facio, que cuenta con un total de 13 facultades, 46 escuelas y 48 unidades de investigación. En esta sede hay un total de $12 \mathrm{SA}$, que son concesionados mediante procesos de contratación administrativa en los cuales median especificaciones técnicas definidas por la administración de la Universidad.

En este contexto, para los SA en los que se efectuó la medición, se tiene establecido un patrón de menú tanto para desayuno como para almuerzo, patrón que debe ser cumplido por el concesionario. Lo anterior implica mantener un cálculo ágil de las proyecciones de venta y conocer el gusto de los comensales por los platillos ofrecidos para determinar la cantidad que se debe producir. Los tamaños de porción para gran parte de los alimentos también son establecidos por la administración de la UCR.

En la tabla 1, se indican los patrones de menú de desayuno y almuerzo para cada uno de los SA con los que se trabajó la medición.
Cabe resaltar que en la medición se contemplaron los alimentos producidos en los SA, aunque en algunos casos presentaban alimentos que no se encontraban dentro del patrón de menú, por ejemplo, empanadas en el desayuno. Es importante conocer el patrón de menú para dimensionar la complejidad operativa de estos SA y las limitaciones que se pueden presentar en cuanto a la producción de alimentos y sus implicaciones en el desperdicio.

Este estudio fue de tipo descriptivo y transversal debido a que describe la variable de desperdicio de alimentos en un periodo de tiempo determinado (entre 2018 y 2019) para algunos de los SA con mayor afluencia de la Sede Rodrigo Facio de la Universidad de Costa Rica. Se tomó una muestra a conveniencia, realizando mediciones en dos SA en el 2018 y 2019. Un tercer SA fue incluido en el estudio en el 2019. La cantidad de SA se definió de acuerdo con la cantidad de recurso humano disponible para realizar las mediciones. La selección de los SA respondió a la capacidad de cada uno de ellos para poder poner una estación de medición, pues algunos tienen limitaciones de espacio en las áreas de producción y servicio.

Estos SA se caracterizan por ofrecer jornada continua desde las 7 a. m. hasta las 7 p. m. y hasta las 8 p. m. en el caso de uno de ellos, de lunes a viernes, con un ciclo de menú variado, de dos semanas para el desayuno y de cinco semanas para el almuerzo, con el mismo patrón de menú para cada tiempo de comida en todos los servicios muestreados. La población de estos SA está conformada por estudiantes, personal docente y administrativo de la UCR y en menor medida atienden visitantes externos. 
Tabla 1. Patrón de menú de los tiempos de comida evaluados para los servicios de alimentos estudiados de la Universidad de Costa Rica

\begin{tabular}{lll}
\hline Tiempo de comida & Patrón de menú & Opciones \\
\hline Combo 1 & Componente principal \\
& Guarnición & Componente principal \\
Combo 2 & Guarnición \\
Fesayuno & Componente principal \\
& Bebidas & Guarnición \\
& 2 opciones \\
& Fresco con azúcar \\
& Fresco sin azúcar \\
Platos fuertes & Bebida caliente \\
& Plato fuerte o componente principal compuesto \\
Acompañamiento & Plato fuerte o componente principal en trozo \\
Guarnición & Plato fuerte o componente principal 3 (plato vegetariano) \\
Ensaladas & Arroz y frijoles, y/o puré según sea el caso \\
(o barra de ensalada con 6 ingredientes) & 1 opción (vegetal) \\
Frescos & 2 opciones \\
Frutas & 1 opción sin azúcar \\
\hline
\end{tabular}

Las mediciones se llevaron a cabo entre la Escuela de Nutrición de la UCR y la Sección Gestión de Servicios Contratados, de la Oficina de Servicios Generales. También, se contó con apoyo logístico de la Unidad de Gestión Ambiental de la Universidad. La metodología utilizada para la cuantificación se basó en la Guía de medición del desperdicio de alimentos en cocinas institucionales y comerciales de 2017, elaborada por la Red Costarricense para la Disminución de la PDA (11).

En dicho documento se establecen las cuatro etapas que se deben realizar para llevar a cabo el proyecto, estas son 1) contacto inicial y elaboración del plan de medición, 2) medición de la PDA, 3) sensibilización y toma de acciones para disminuir pérdidas y 4) verificación de mejoras a través de nuevas mediciones. Esta investigación abarcó las etapas 1 y 2 . Para en un estudio posterior, se propone generar las líneas de acción para los puntos 3 y 4 .

Con respecto al formulario de recolección de datos establecido en la guía de medición (11), se hicieron ajustes para organizarlo por áreas operativas del servicio (almacenamiento, producción y servicio) de acuerdo con las que se tienen definidas en Ios SA de la UCR, esto debido a que en esta guía se brinda un ejemplo de formulario genérico, pues se considera que debe ser aplicable a diferentes tipos de SA, aunque se especifica que en algunos casos podría requerir ajustes.

La toma de datos se realizó por parte de estudiantes de cuarto año de la carrera de Nutrición mediante las metodologías de pasantía o práctica extramuros en SA, siempre supervisadas por las contrapartes profesionales en nutrición de la Escuela de Nutri- 
ción y la Sección Gestión de Servicios Contratados de la UCR. Debido a lo anterior, se realizó una serie de sesiones de capacitación para estandarizar el proceso de cuantificación y asegurar la comprensión de los lineamientos de la Guía de medición del desperdicio de alimentos en cocinas institucionales y comerciales (11).

También se estandarizaron los términos con los que se iba a trabajar, estableciendo que el desperdicio es cuando un alimento, destinado para consumo humano, fue desechado por el consumidor o los operarios del servicio. La fracción comestible es la porción que se puede consumir de un alimento con base en 100 gramos, por lo tanto, no se toman en cuenta los huesos, las espinas, entre otros. Se entendió como desgaste la fracción no comestible del alimento y se tomó como referencia la fracción comestible del Instituto de Nutrición de Centroamérica y Panamá (INCAP) (12); así como los factores de conversión, que se aplican para obtener el cambio de peso de alimentos crudos a cocidos y viceversa (13).

Se realizó una prueba piloto en un servicio de alimentación que no formaba parte de la muestra y posterior a esta prueba se hicieron las adaptaciones al formulario de recolección de información indicados en la guía de medición, esto para adaptarlos a las áreas operativas con que cuentan los SA de la UCR. Esta prueba piloto también sirvió para mejorar aspectos logísticos en cuanto a la recolección y separación de residuos en plato.

El presente estudio se realizó en tres SA del campus Rodrigo Facio de la Universidad de Costa Rica, en los siguientes periodos de muestreo: SA N1 2018 y 2019, SA N2 2018 y 2019 y SA N3 2019, entre los meses de mayo a junio en cada año. Se realizaron tres mediciones, siempre en día miércoles, en cada servicio, con un total de 15 mediciones, con base en la guía antes mencionada, y se aplicó un análisis de varianza (ANOVA).

Las mediciones se realizaron de 7 a. m. a 3 p. m., en tres miércoles (debido a la disponibilidad del recurso humano), en turnos entre estudiantes y profesionales en nutrición y se abarcaron los tiempos de comida de desayuno, merienda de la mañana y almuerzo (que son los tiempos principales en estos servicios). Cabe destacar que debido al traslape de horario que se da entre el desayuno y la merienda de la mañana se decidió juntar estos tiempos de comida y considerarlos ambos como desayuno; por lo tanto, cuando se indique desayuno a lo largo de este documento, este por defecto contiene los datos de merienda de la mañana.

En las áreas de preparación se cuantificó la masa en $\mathrm{kg}$ de los alimentos, antes y después de los procesos y se evaluó si el residuo correspondía a desgaste (fracción no comestible) o a desperdicio (alimento o parte del alimento consumible). En el área de bodega se cuantificó la masa en $\mathrm{kg}$ de alimentos que correspondía a desperdicio. También se midió el desperdicio en los platos de comida de los comensales cuando estos terminaron de comer; para lo cual se identificaron con anterioridad las porciones que correspondían a desgaste propio del alimento, por ejemplo, huesos; por lo tanto, no se consideraron como desperdicio.

Con base en las mediciones, se cuantificó un porcentaje de desperdicio por área y una cuantificación total del desperdicio diario del SA. Para efectos de este estudio, se entiende desperdicio de alimentos como la cantidad de alimentos que se descartó, pero su descarte se pudo haber evitado controlando aspectos como rotación de inventarios, tamaños de porción, manipulación adecuada de alimentos y técnicas culinarias adecuadas. En la figura 1 se puede observar el diagrama de entradas y salidas. 


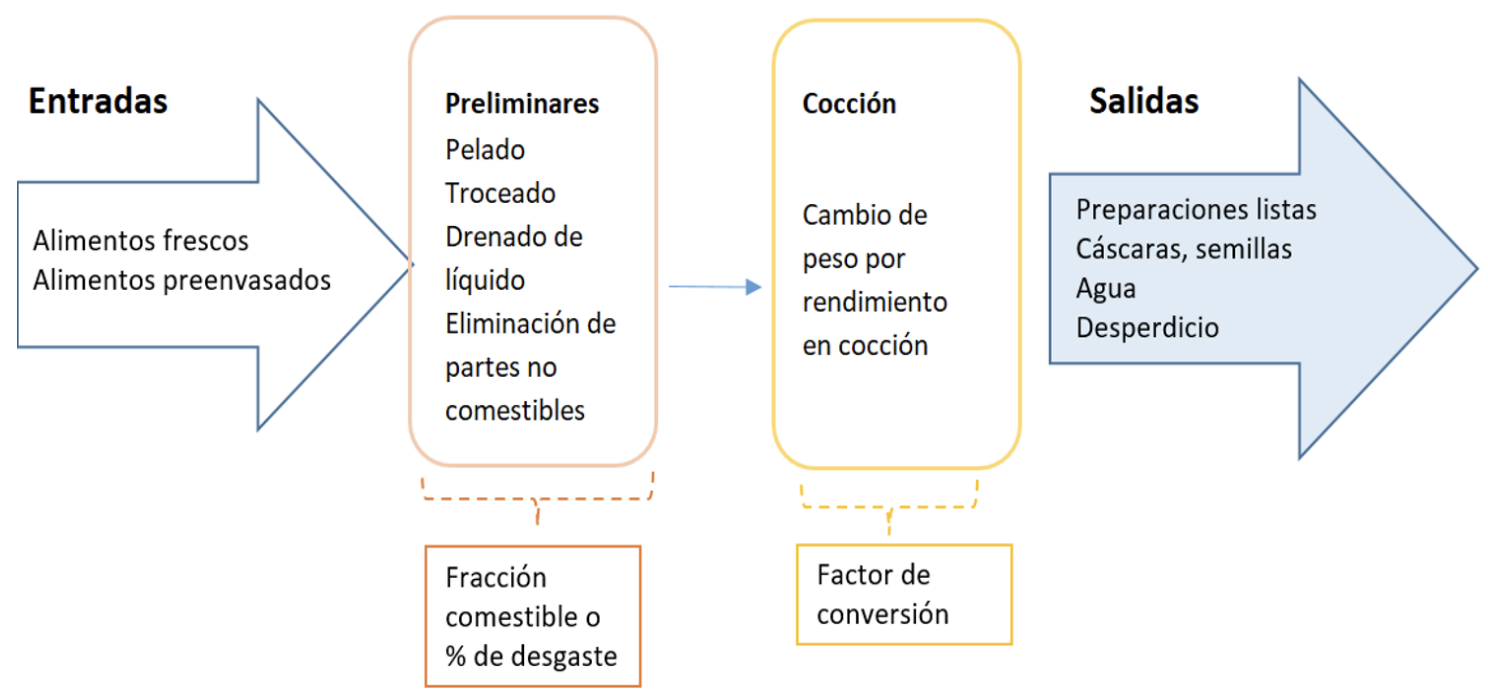

Figura 1. Diagrama de entradas y salidas consideradas para determinar el porcentaje de desperdicio de alimentos en los servicios de alimentación de la Universidad de Costa Rica.

Los materiales y equipos utilizados para la medición fueron balanzas de recibo marca Torrey capacidad $40 \mathrm{~kg}$ y sensibilidad $5 \mathrm{~g}$, y balanza Torrey capacidad $50 \mathrm{~kg}$ y sensibilidad $9 \mathrm{~g}$. Además, fueron necesarios otros suministros como guantes, bata (gabacha), cofia, bolsas plásticas, cuadernos, lapiceros y baldes para contener los alimentos desperdiciados.

Se revisó con anterioridad el menú de desayuno y almuerzo que se ofrecería el día de la medición en cada servicio de alimentación, mediante la aplicación móvil y sitio web SODA UCR y se confirmó con los concesionarios, para garantizar que no hubiera cambios y conocer el plato especial del día. Esto con el fin de tener mayor claridad en el desgaste (fracción no comestible) de los alimentos que se iban a ofrecer, así como conocer si era necesario considerar otros requerimientos logísticos, como el manejo de huesos, entre otros.
Una vez concluido el periodo de mediciones, se procedió a organizar la información en cuadros en el programa Excel ${ }^{\circledR}$. Para el procesamiento de los datos, se consideraron los porcentajes de fracción comestible de la Tabla de Composición de Alimentos del INCAP. El análisis de los datos del desayuno y del almuerzo se realizaron por aparte. Se procedió a determinar el desperdicio de alimentos en $\mathrm{kg}$ de las áreas de almacenamiento, área de producción, barra de servicio y plato servido; posteriormente, se sumaron estos datos, se compararon con la producción total de sólidos en $\mathrm{kg}$ de cada servicio y se reportó el porcentaje de toda la producción que fue desperdiciado. Al final los datos de líquidos no se presentaron.

El porcentaje de desperdicio de alimentos por servicio de alimentación se obtuvo con relación a la producción total de alimentos para cada SA por los tiempos de comida, estudiados mediante las siguientes ecuaciones descritas por Rojas-Vargas et al. (14): 
[1] Producción diaria total (PDT)

$\mathrm{PDT}=\sum(\mathrm{Pi}-\mathrm{Pf})$

Donde $\mathrm{Pi}=$ peso del producto inicial $(\mathrm{kg})$, $\mathrm{Pf}=$ peso del producto final $(\mathrm{kg})$

[2] Porcentaje de PDA con respecto a la producción diaria (\%PDA PD) en cada día

$\% P D A P D=F / P D * 100$

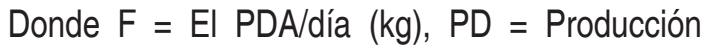
diaria/ día $(\mathrm{kg})$

\section{Análisis estadístico}

El análisis estadístico de los datos se realizó con el software JMP ${ }^{\circledR} 9$. Se efectuó un ANOVA de una vía para determinar las diferencias significativas entre los porcentajes de desperdicio por área, la producción total en $\mathrm{kg}$, en los dos SA medidos en 2018 y en los tres SA medidos en 2019. En los casos en los que se encontró diferencia significativa $(p<0,05)$, se realizó una comparación de todos los pares usando t de Student.

\section{RESULTADOS}

En la tabla 2 se muestra el resumen de los datos obtenidos en la cuantificación de desperdicio de alimentos en los SA seleccionados. Se muestran datos totales promedio de las tres mediciones para producción total y porcentajes obtenidos de desperdicio de alimentos por áreas operativas (almacenamiento, producción y servicio), así como las respectivas diferencias identificadas mediante el análisis estadístico.

En cuanto a la producción promedio entre los SA no se observaron diferencias significativas entre los datos medidos $(p=0,1467)$ durante los dos años. Lo anterior indica que la producción en estos SA es comparable y no varió entre el 2018 y el 2019. No se encontraron diferencias significativas $(p>0,05)$ en los SA evaluados para los porcentajes de desperdicio de alimentos en las áreas operativas de almacenamiento $(p=0,1293)$ ni en la producción de alimentos $(\mathrm{p}=0,7718)$. Los mayores porcentajes de desperdicio se observaron en el área de servicio: en barra, platos de desayuno y platos de almuerzo (Tabla 2).

Tabla 2. Promedios de producción total de alimentos y porcentaje de desperdicio de alimentos con respecto a la producción total por área para el tiempo del desayuno y del almuerzo

\begin{tabular}{|c|c|c|c|c|c|c|}
\hline \multirow{4}{*}{ Área de medición } & \multicolumn{5}{|c|}{ Año } & \multirow[b]{3}{*}{ Valor de p† } \\
\hline & \multicolumn{2}{|c|}{2018} & \multicolumn{3}{|c|}{2019} & \\
\hline & SA N1 & SA N2 & SA N1 & SA N2 & SA N3 & \\
\hline & $\mathrm{n}=3$ & $\mathrm{n}=3$ & $n=3$ & $n=3$ & $\mathrm{n}=3$ & \\
\hline Producción total por día sólida (kg) & $382,7^{a}$ & $327,4^{\mathrm{a}}$ & $327,9^{a}$ & $478,0^{a}$ & $382,7^{a}$ & 0,1467 \\
\hline Desperdicio en área de almacenamiento (\%) & $0,1^{\mathrm{a}}$ & $2,4^{\mathrm{a}}$ & $0,0^{\mathrm{a}}$ & $0,4^{\mathrm{a}}$ & $0,0^{\mathrm{a}}$ & 0,1293 \\
\hline Desperdicio en el área de producción (\%) & $2,0^{\mathrm{a}}$ & $1,2^{\mathrm{a}}$ & $0,8^{\mathrm{a}}$ & $0,7^{\mathrm{a}}$ & $1,3^{\mathrm{a}}$ & 0,7718 \\
\hline Desperdicio en barra de servicio (\%) & $3,5^{\mathrm{ab}}$ & $5,4^{a}$ & $5,5^{\mathrm{a}}$ & $1,2^{b}$ & $1,9^{b}$ & 0,0197 \\
\hline Desperdicio platos servidos desayuno (\%) & $4,5^{\mathrm{a}}$ & $1,8^{\mathrm{ab}}$ & $0,7^{b}$ & $0,9^{b}$ & $0,4^{b}$ & 0,0075 \\
\hline Desperdicio platos servidos almuerzo (\%) & $6,6^{\mathrm{a}}$ & $6,3^{\mathrm{ab}}$ & $4,5^{\mathrm{abc}}$ & $3,8^{\mathrm{bc}}$ & $2,9^{c}$ & 0,0362 \\
\hline $\begin{array}{l}\text { Total de desperdicio diario con respecto a } \\
\text { masa de producción sólidos (\%) }\end{array}$ & $16,6^{a}$ & $15.0^{\mathrm{a}}$ & $11,5^{\mathrm{ab}}$ & $7,0^{b}$ & $6,5^{b}$ & 0,0054 \\
\hline
\end{tabular}

*No se cuantificó producción total de líquidos, por eso se presenta en kg y no porcentaje.

†Valor de $p$ según la prueba ANOVA. En cada fila se presenta con letra diferente las que tienen diferencias significativas según la prueba t de Student $(p<0.05)$. Letras iguales indican que no hay diferencia $(p>0,05)$. 
Se observaron diferencias significativas para el porcentaje de desperdicio de alimentos en el área operativa de servicio, que comprende desperdicio en barra de servicio $(p=0,0197)$, en plato de desayuno $(p=0,0075)$ y en platos de almuerzo $(p=0,0362)$. En el desperdicio en barra se observó que hay diferencia en el SA N2 entre la medición del 2018 y la del 2019, puesto que, para este servicio, el desperdicio en esta área disminuyó significativamente en el 2019. Además, se pudo evidenciar que entre los tres servicios medidos en el 2019 el N1 tenía mayor desperdicio en barra de servicio con respecto a los otros dos.

En cuanto al desperdicio de platos servidos en el desayuno, se observó que entre los SA medidos en el 2019 no había diferencia significativa. Aunque sí se encontró una reducción significativa en el desperdicio en platos servidos al desayuno para el SA N1 en la medición de 2019 en comparación con la de $2018(p<0,05)$. Del porcentaje de desperdicio en platos servidos al almuerzo se observó que en general es mayor al de platos servidos en el desayuno.
Con respecto al desperdicio total en la producción de sólidos, se encontró que entre los dos SA medidos en el 2018 no había diferencia significativa. Se observó una disminución significativa en el porcentaje de desperdicio total entre las mediciones del 2018 y del 2019 para el SA N2 (Tabla 2). En la figura 2 se puede observar el promedio y el porcentaje del desperdicio total para cada medición realizada y en la figura 3 se muestra el aporte porcentual de desperdicio de cada área al total del desperdicio.

\section{DISCUSIÓN}

En este tipo de SA las preparaciones pasan por un flujo de proceso de los alimentos característico (figura 4), el cual se basa en la legislación costarricense (15). Estos flujos están directamente relacionados con el desperdicio de alimentos y se deben considerar para efectos de la medición. En cada etapa los alimentos sufren modificaciones que pueden derivar en desperdicios, desde el pelado y troceado hasta una manipulación inadecuada que implique tener que desechar el producto (16).

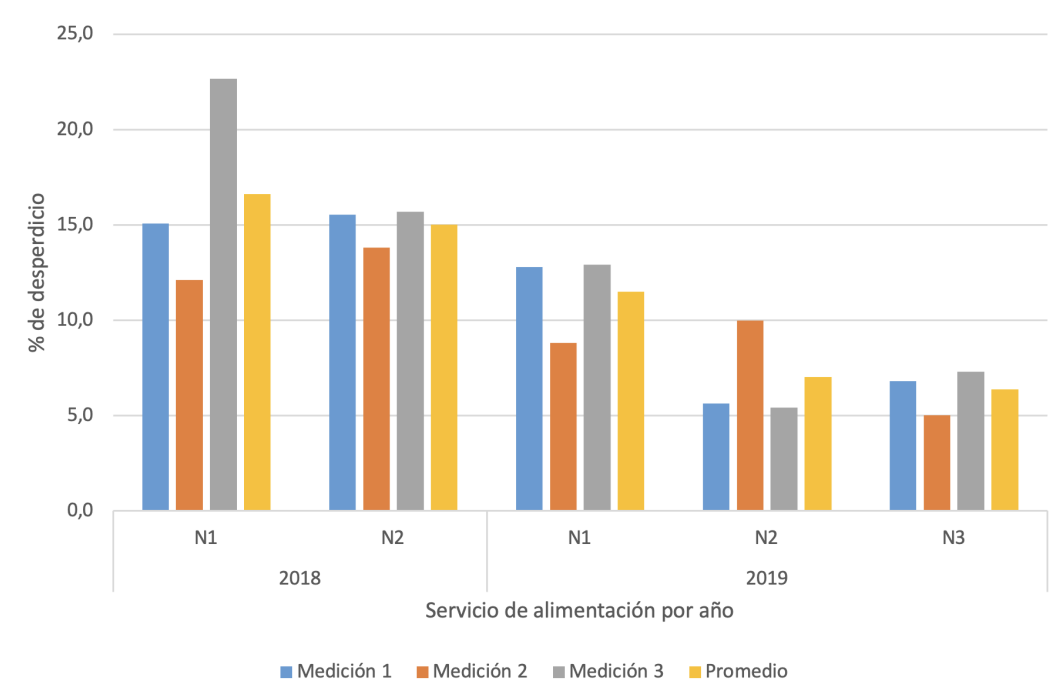

Figura 2. Cuantificación de desperdicio de alimentos en porcentaje con respecto a la producción total. 


\section{Porcentaje del desperdicio total que representa cada una de las áreas}

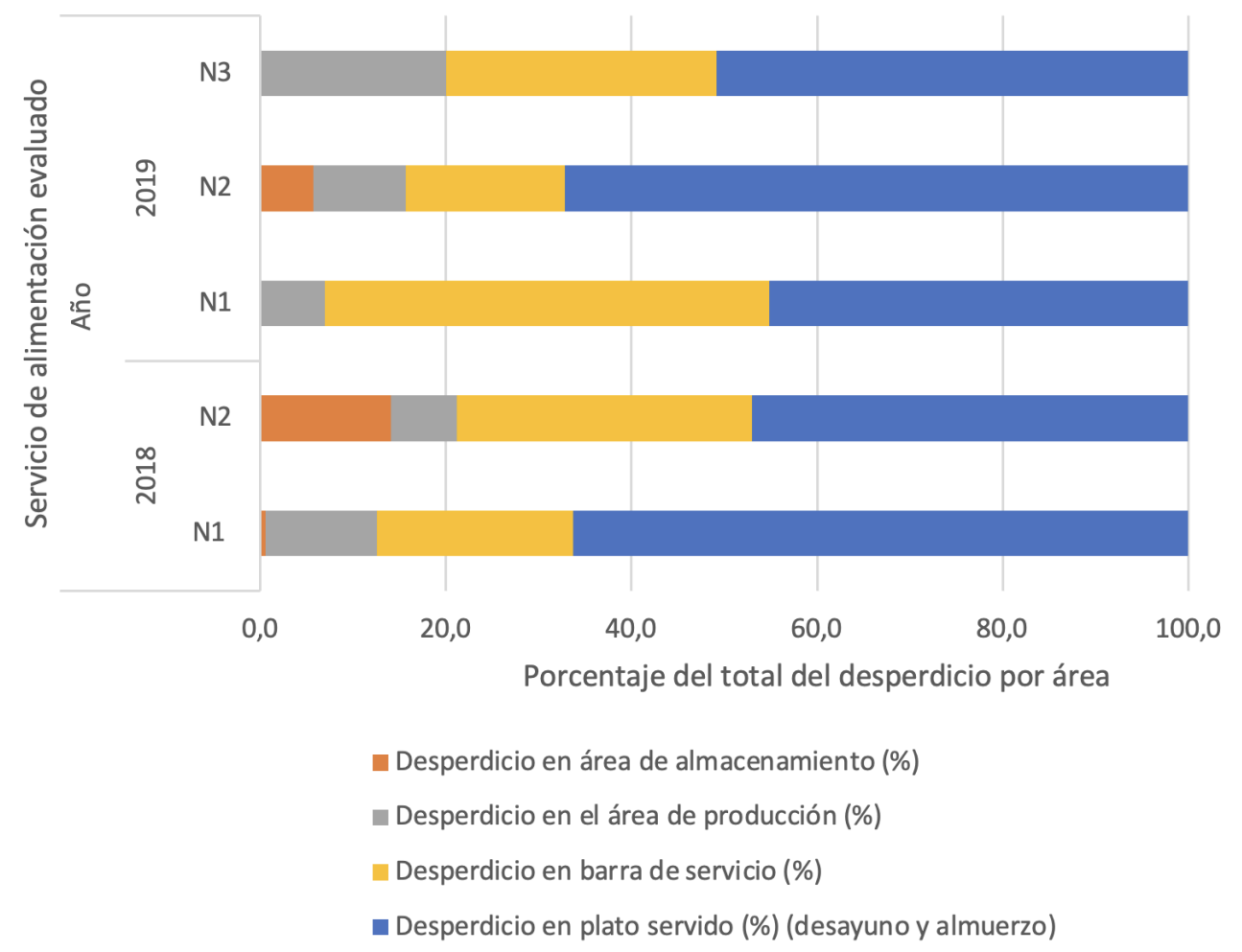

Figura 3. Porcentaje de desperdicio de cada área con respecto al porcentaje total.
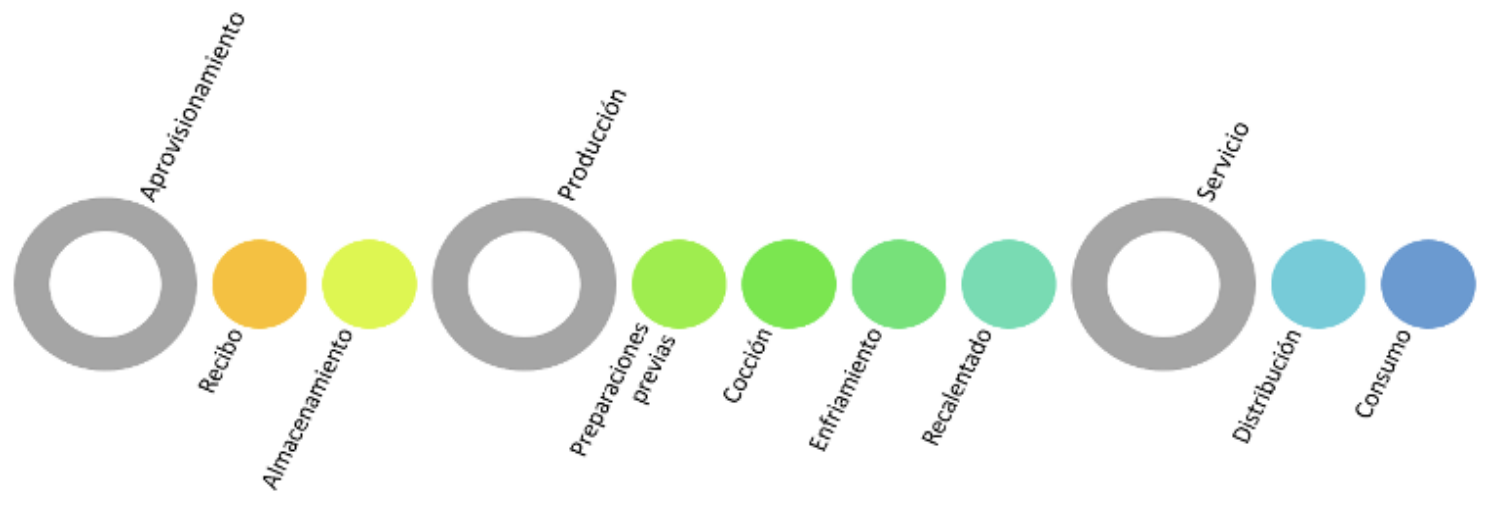

Figura 4. Flujo para el proceso de los alimentos en los servicios de alimentación de la UCR. 
La medición de residuos y desperdicios es un tema relativamente nuevo en Costa Rica; sin embargo, ya existen mediciones realizadas en otros SA que arrojan datos de desperdicio que oscilan entre el 5 y el $23 \%$, según el tipo de operación de la que se trate. En el caso de este estudio, el desperdicio total en todos los SA evaluados estuvo dentro de este rango.

El desperdicio total está compuesto por los desperdicios generados en el área de bodegas (del 2 al $20 \%$ ), preparación (del 4 al $30 \%$ ) y en residuos de platos servidos (del 29 al $81 \%$ ), estos datos son congruentes con otros estudios a nivel internacional en SA $(11,17,18)$. En el caso del presente estudio, en todas las áreas operativas -almacenamiento (bodegas), producción (preparación) y servicio-, se observa que el porcentaje en forma general en los SA evaluados es inferior a lo descrito por los estudios mencionados anteriormente.

Los SA presentan un radio pequeño de m²; por lo tanto, los usuarios pueden decidir a cuál servicio asistir para consumir sus alimentos. Adicionalmente, la Sección Gestión de Servicios Contratados logró generar una aplicación móvil y un sitio web, en coordinación con el Centro de Informática de la Universidad, en los que se publican los menús de todos los SA de la UCR y los usuarios los pueden consultar en cualquier momento para conocer de manera previa el menú, esto es una muy buena acción en cuanto a servicio al usuario; no obstante, representa un reto en la programación de producción para los concesionarios.

Para el área de almacenamiento de los SA analizados en la UCR, el desperdicio fue mucho menor a lo reportado en los otros estudios, en los que se indica hasta un $13 \%$ del desperdicio total identificado en esta área $(14,19)$. Lo anterior se atribuye a un buen control de la rotación de la materia prima en bodega, por ejemplo, la estrategia de "Primero en entrar, primero en salir", con el fin de permitir el flujo adecuado de los productos más próximos a vencerse (20).

En el caso de los porcentajes de desperdicio en la producción, no hubo diferencia significativa entre los SA evaluados. Al comparar con lo descrito en otros estudios, los porcentajes de desperdicio en producción del presente estudio fueron menores a los reportados en la literatura, con valores de aporte al total de desperdicio de entre el 7 y el $20 \%$, ninguno cercano al $30 \%$ descrito en estudios anteriores (11). También son porcentajes de desperdicio bajos en esta área en comparación con el 29,34 \% que se reporta en otra institución educativa de una universidad pública de Costa Rica (14).

Por otra parte, el desecho que se reporta con mayores cantidades en diversos estudios es el proveniente de los residuos en platos servidos, de entre el 29 y el $81 \%$, e incluso lo reportado en otros estudios con SA similares de hasta un $69 \%$ $(10,14,19)$. En este caso, los resultados obtenidos presentan una leve disminución con respecto a lo que indican los estudios; sin embargo, el mayor porcentaje de desperdicio es aportado por el desperdicio en platos servidos (45 a $67 \%$ ). Esto concuerda con lo encontrado en otro estudio de otra institución de educación superior en Costa Rica, en el que el residuo en plato servido fue el que presentó el mayor desperdicio (14) con condiciones de tipo de servicio similares a las presentes en este estudio.

Así mismo, los datos del presente estudio, respecto al desperdicio y al porcentaje de producción total, son menores a otros estudios que reportan $25 \%$ con respecto a la producción total en SA universitarios; en otros SA, como restaurantes, cafeterías, lugares de cuido, estuvo entre el 17 y el $28 \%$ (21). Con respecto a otras instituciones 
educativas de Costa Rica, el promedio de desperdicio de cinco instituciones fue de 11,3\% (22) con respecto a la producción total. Para el 2019, el promedio de los tres SA evaluados en la UCR fue de $8,3 \%$, es decir, más bajo que el promedio del resto de instituciones similares del país.

Un mayor desperdicio en plato se podría atribuir a poca aceptabilidad por parte de los usuarios en las opciones del día (23), que las porciones servidas sean muy grandes (24) o que incluso los hábitos y costumbres cambian a través del tiempo; por ejemplo, consumir menos arroz, considerando que puede ser un alimento que cause exceso de peso (25).

Algunos estudios mencionan que los patrones de menú y la oferta por grupos de alimentos también se relacionan con el desperdicio de alimentos (26). El patrón de menú de los SA de la UCR es adecuado a la dieta usual costarricense, además de ser balanceado a las necesidades de la población, por lo que se considera que esto puede ser un factor fundamental para que se presente un desperdicio un poco menor al descrito en otros estudios $(6,27)$.

También, variables como las opciones de comida y la calidad de los alimentos que percibe el usuario son factores que afectan la cantidad de desperdicio (28). Otras razones que se han encontrado en estudios similares sobre las razones del desperdicio de comida han sido que los alimentos no gustaron, el tiempo limitado o la falta de hambre (29).

De igual forma, el tamaño de porción es un factor determinante en el desperdicio en plato $(26,30)$, esto es difícil de manejar en los SA de la UCR, porque, si bien se atiende sobre todo a adultos, los patrones de consumo difieren entre estudiantes, trabajadores (docentes y administrativos) y otros grupos de personas que visitan estos servicios, así como las conocidas diferencias en cuanto a requerimientos nutricionales entre hombres y mujeres.

Establecer un tamaño de porción que supla los requerimientos y necesidades de toda la población atendida es complejo; no obstante, impulsa a hacer una revisión para mejorar los tamaños de porción y también a sensibilizar a los usuarios sobre la cantidad y tipo de alimentos que piden en estos SA. Se ha visto que campañas de sensibilización contribuyen a disminuir el desperdicio de alimentos en los SA (31).

Otro factor que impacta en el desperdicio en plato en estos SA es el hecho de que hay opciones que se ofertan como "combos", es decir, comidas que incluyen varios componentes (32). Se observó que algunos usuarios que tenían grandes cantidades de desperdicio en plato mencionaban que pedían completo el plato porque igual se los iban a cobrar, aunque no se lo iban a comer todo. Otras personas mencionaron que, por las dinámicas del servicio, cuando llegaban a la parte donde les servían el plato ya les habían incluido alimentos básicos (como arroz y frijoles) y no tenían opción de indicar que querían una menor cantidad 0 que de todo lo que se ofrecía no querían algún alimento. Lo anterior es congruente con lo reportado en estudios en los que se habla del efecto del "buffet" en el desperdicio (30).

Los índices bajos de desperdicio en las áreas de almacenamiento y producción se pueden deber a los márgenes de utilidad con los que trabajan los SA de la UCR. El precio de venta del plato es uno de los aspectos más importantes en el proceso de contratación; por lo tanto, se compite entre otros factores por precio de plato, esto genera que los interesados en operar uno de estos servicios tiendan a ofertar un precio muy competitivo (no ruinoso). Se debe destacar que en la licita- 
ción se establece una utilidad no menor del $10 \%$; sin embargo, debido a que los precios ofertados son de bajo costo para que sean accesibles para la población, los márgenes de utilidad podrían ser relativamente bajos. Debido a lo anterior, no se pueden desperdiciar insumos, en este caso alimentos.

Los resultados obtenidos muestran la importancia de determinar líneas de acción para la sensibilización y campañas de disminución de PDA. Por ejemplo, el desperdicio en la zona de producción puede deberse a varios factores como la capacitación del personal, que eventualmente podría estar realizando técnicas de corte que generaran un aumento en la cantidad de producto comestible no aprovechada (5).

Al analizar el desperdicio total con respecto a la producción, este se observa por debajo de lo esperado, ya que de forma general se esperaría que ronde entre un 17 y un $30 \%(6,14,27,33)$. Para el 2019 se observa una reducción del $55 \%$ del desperdicio de alimentos con respecto al 2018, pasando de un $15,5 \%$ en 2018 a un 8,3\% en el 2019 , en promedio, con respecto a la producción total.

Como se mencionó en los resultados, se logró cuantificar una reducción estadísticamente significativa entre el 2018 y el 2019 para el SA N2, esto se atribuye a que, a pesar de no haber realizado una intervención como tal, probablemente las mediciones del 2018 tuvieron un efecto sensibilizador tanto en los concesionarios y el personal del SA como en los usuarios; además, se ha visto que la capacitación en el personal de cocina puede generar disminuciones en el desperdicio de alimentos (34). También, antes de las mediciones del 2019, se realizó una capacitación con los concesionarios de los SA de la UCR con respecto a la guía de medición, esto pudo haber tenido un impacto en el porcentaje total de desperdicio de ese año.

Otro aspecto que se considera relevante es que, si bien no se realizó una cuantificación por tipo de alimento en el desperdicio en plato, por medio de la observación se logró identificar que lo que más se desperdiciaba era el arroz, la ensalada y el fresco, por lo que es de suma importancia reevaluar el tamaño de porción establecida para estos alimentos, así como las combinaciones que se realicen con el plato fuerte. Se han desarrollado métodos de cuantificación de desperdicio mediante la observación (35), esto puede ser una oportunidad para futuras investigaciones, debido a que medir de forma directa alimentos por separado es muy complejo y requiere muchos recursos tanto humanos como materiales.

\section{AGRADECIMIENTOS}

A los concesionarios y personal de los SA por su anuencia y colaboración. A las estudiantes de la Escuela de Nutrición que participaron en las mediciones. Al profesor Eric Wong González por su ayuda en la parte de análisis de datos.

\section{CONFLICTO DE INTERESES}

No hay ningún conflicto de intereses, debido a que esta investigación se realizó con recursos de la UCR y no responde a ningún interés particular.

\section{FINANCIACIÓN}

No hubo un apoyo financiero extra para realizar la investigación. El recurso utilizado fue tiempo laboral de la UCR. 


\section{-Referencias}

1. ONU. Informe de los objetivos del desarrollo sostenible. [Citado diciembre de 2019]. Disponible en: https://unstats.un.org/ sdgs/report/2019/The-Sustainable-Development-Goals-Report-2019 Spanish.pdf

2. Beretta $\mathrm{C}$, Hellweg S. Potential environmental benefits from food waste prevention in the food service sector. Resour Conserv Recycl [Internet]. 2019;147(March):169-78. https://doi.org/10.1016/i.resconrec.2019.03.023

3. Al-Rumaihi A, McKay G, Mackey HR, Al-Ansari T. Environmental impact assessment of food waste management using two composting techniques. Sustain. 2020;12(4). https://doi.org/10.3390/su12041595

4. FAO. Global food losses and food waste - Extent, causes and prevention. Food Loss and Food Waste: Causes and Solutions. Rome; 2011. [Citado diciembre de 2019]. Disponible en: http://www.fao.org/3/a-i2697e.pdf

5. Dhir A, Talwar S, Kaur P, Malibari A. Food waste in hospitality and food services: A systematic literature review and framework development approach. J Clean Prod [Internet]. 2020;270:122861. https://doi.org/10.1016/j.jclepro.2020.122861

6. Silvennoinen K, Nisonen S, Pietiläinen O. Food waste case study and monitoring developing in Finnish food services. Waste Manag. 2019;97:97-104. https://doi.org/10.1016/j.wasman.2019.07.028

7. FAO. Food losses and waste in Latin America and the Caribbbean. 2014;(July):8. [Citado diciembre de 2019]. Disponible en: http://www.fao.org/3/i4655e/i4655e.pdf

8. Costa Rica. Red Costarricense para Disminuir la Pérdida y Desperdicio de Alimentos. [Citado diciembre de 2019]. Disponible en: https://www.tec.ac.cr/red-costarricense-disminucion-perdidas-desperdicios-alimentos

9. Costa Rica. Red Costarricense de Instituciones Educativas Sostenibles: retos y Oportunidades 2010-2017 [Citado diciembre de 2019]. Disponible en: http://docplayer.es/94770959-Red-costarricense-de-instituciones-educativas-sostenibles-retos-yoportunidades.html

10. Ellison B, Savchenko O, Nikolaus CJ, Duff BRL. Every plate counts : Evaluation of a food waste reduction campaign in a university dining hall. Resour Conserv Recycl. 2019;144:276-84. https://doi.org/10.1016/j.resconrec.2019.01.046

11. Red Costarriense para disminuir pérdida y desperdicio de alimentos. Guia de medición del desperdicio de alimentos en cocinas institucionales y comerciales. Cartago: Editorial Tecnológica de Costa Rica; 2017, 40 pp. https://www.tec.ac.cr/sites/ default/files/media/doc/2_guia_medicion_cocinas_web.pdf

12. INCAP. Tabla de Composición de Alimentos de Centroamérica. 2012. 128 pp. [Citado diciembre de 2019]. Disponible en: http://www.incap.int/mesocaribefoods/dmdocuments/tablacalimentos.pdf

13. Murillo S, Ulate E. Tabla de composición de alimentos y de pesos para Costa Rica. San José; 1984. [Citado diciembre de 2019]. Disponible en: https://www.binasss.sa.cr/opacms//media/digitales/Tabla\%20de\%20composici\%C3\%B3n\%20de\%20 alimentos\%20y\%20pesos\%20para\%20C.R.pdf

14. Rojas-Vargas J, Monge-Fernández Y, Fernández-Hidalgo K. Desperdicios de alimentos (DA) en sodas concesionadas de una Universidad Pública en Heredia, Costa Rica. Rev Tecnol en Marcha. 2020;33:152-64. https://doi.org/10.18845/tm.v33i1.5029

15. Gobierno de Costa Rica. Reglamento para los Servicios de Alimentación al Público. Costa Rica; 2012 p. 1-86. [Citado diciembre de 2019]. Disponible en: http://www.pgrweb.go.cr/scij/Busqueda/Normativa/Normas/nrm_texto_completo.aspx?param1= NRTC\&nValor1=1\&nValor2=73436\&nValor3=90132\&strTipM=TC

16. Hennchen B. Knowing the kitchen: Applying practice theory to issues of food waste in the food service sector. J Clean Prod [Internet]. 2019;225:675-83. https://doi.org/10.1016/j.jclepro.2019.03.293 


\section{Cuantificación de desperdicio de alimentos en servicios de alimentación}

17. Eriksson M, Lindgren S, Persson C. Mapping of food waste quanti fi cation methodologies in the food services of Swedish municipalities. Resour Conserv Recycl [Internet]. 2018;137(June):191-9. https://doi.org/10.1016/j.resconrec.2018.06.013

18. Eriksson M, Persson Osowski C, Björkman J, Hansson E, Malefors C, Eriksson E, et al. The tree structure - A general framework for food waste quantification in food services. Resour Conserv Recycl [Internet]. 2018;130(November 2017):140-51. https://doi.org/10.1016/j.resconrec.2017.11.030

19. Eriksson M, Persson Osowski C, Malefors C, Björkman J, Eriksson E. Quantification of food waste in public catering services - A case study from a Swedish municipality. Waste Manag [Internet]. 2017;61:415-22. http://dx.doi.org/10.1016/j.wasman.2017.01.035

20. Ahmed MO. The operation of food production in egyptian university hospitals. Int J Hosp Tour Syst. 2018;11(1):47-59. Disponible en: http://www.publishingindia.com/ijhts/24/the-operation-of-food-production-in-egyptian-university-hospitals/659/4619/

21. Silvennoinen K, Heikkilä L, Katajajuuri J, Reinikainen A. Food waste volume and origin : Case studies in the Finnish food service sector. Waste Manag. 2015;46:140-5. http://dx.doi.org/10.1016/j.wasman.2015.09.010

22. Rojas-Vargas J, Monge-Fernández Y, Jiménez-Morales MF, Arguedas-Camacho M, Hidalgo-Viquez C, Peña-Vásquez M, et al. servicios de alimentación de instituciones Food loss and waste in food services from educational institutions in Costa Rica. Tegnología en Marcha. 2021;34(2):187-96. https://doi.org/10.18845/tm.v34i2.4854

23. Valero Díaz A, Caracuel García Á. Evaluación de factores influyentes sobre el desecho de alimentos por parte de pacientes procedentes de diferentes recintos hospitalarios. Nutr Hosp. 2013;28(2):419-27. https://doi.org/10.3305/nh.2013.28.2.6262

24. Ravandi B, Jovanovic N. Impact of plate size on food waste: Agent-based simulation of food consumption. Resour Conserv Recycl [Internet]. 2019;149(July 2019):550-65. https://doi.org/10.1016/j.resconrec.2019.05.033

25. Wu Y, Tian X, Li X, Yuan H, Liu G. Characteristics, influencing factors, and environmental effects of plate waste at university canteens in Beijing, China. Resour Conserv Recycl. 2019;149:151-9. https://doi.org/10.1016/j.resconrec.2019.05.022

26. Lassen AD, Christensen LM, Spooner MP, Trolle E. Characteristics of canteens at elementary schools, upper secondary schools and workplaces that comply with food service guidelines and have a greater focus on food waste. Int J Environ Res Public Health. 2019;16(7):1115. https://doi.org/10.3390/ijerph16071115

27. Papargyropoulou E, Steinberger JK, Wright N, Lozano R, Padfield R, Ujang Z. Patterns and causes of food waste in the hospitality and food service sector: Food waste prevention insights from Malaysia. Sustain. 2019;11(21):6016. https://doi.org/10.3390/su11216016

28. Lebersorger S, Schneider F. Discussion on the methodology for determining food waste in household waste composition studies. Waste Manag.2011;31(9-10):1924-33. http://dx.doi.org/10.1016/j.wasman.2011.05.023

29. Painter K, Thondhlana G, Wei H. Food waste generation and potential interventions at Rhodes University, South Africa. Waste Manag. 2016;56:491-7. http://dx.doi.org/10.1016/j.wasman.2016.07.013

30. Abdelaal AH, McKay G, Mackey HR. Food waste from a university campus in the Middle East: Drivers, composition, and resource recovery potential. Waste Manag. 2019;98:14-20. https://doi.org/10.1016/j.wasman.2019.08.007

31. Septianto F, Kemper JA, Northey G. Thanks, but no thanks: The influence of gratitude on consumer awareness of food waste. J Clean Prod. 2020;258:120591. https://doi.org/10.1016/j.jclepro.2020.120591

32. Pinto RS, Pinto RMDS, Melo FFS, Campos SS, Cordovil CM. A simple awareness campaign to promote food waste reduction in a University canteen. Waste Manag. 2018;76(2018):28-38. https://doi.org/10.1016/j.wasman.2018.02.044

33. Ahmed S, Shanks CB, Lewis M, Leitch A, Smith EM, Hess D. Meeting the food waste challenge in higher education challenge. Int J Sustain High Educ.2018;19(6):1075-94. https://doi.org/10.1108//JSHE-08-2017-0127 
34. Goonan S, Mirosa M, Spence H. Systems-practice framework: An integrated approach for foodservice management. Nutr Diet. 2015;72(1):81-90. https://doi.org/10.1111/1747-0080.12114

35. Martins ML, Cunha LM, Rodrigues SSP, Rocha A. Determination of plate waste in primary school lunches by weighing and visual estimation methods: A validation study. Waste Manag [Internet]. 2014;34(8):1362-8. http://dx.doi.org/10.1016/j.wasman.2014.03.020 